\title{
CAN ACROCHORDONS BE A MARKER OF METABOLIC SYNDROME?
}

\author{
Devyani De1, Mallika Biswas², Dayamay Pal3 ${ }^{3}$ Tarun Biswas ${ }^{4}$ \\ ${ }^{1}$ Assistant Professor, Department of Biochemistry, CNMC. \\ ${ }^{2}$ Associate Professor, Department of Biochemistry, NRSMC. \\ ${ }^{3}$ Assistant Professor, Department of Biochemistry, CNMC. \\ ${ }^{4}$ Junior Resident, Department of Biochemistry, MMC.
}

\section{ABSTRACT}

\section{BACKGROUND}

Acrochordon or fibroepithelial polyp known as skin tags are one of the most common benign skin conditions. It consists of skin projecting from the surrounding skin usually occurring in the eyelids, neck and axilla. The aetiology of acrochordons is still unknown. They usually vary in diameter and are skin coloured. The incidence of obesity in India is increasing at an alarming rate. Less physical activity amongst both children and adults are leading to it. The aim of the present study is to find out if there is any association of acrochordons with blood glucose, HbA1c, lipid profile and BMI in outdoor patients selected from the Department of Dermatology, Calcutta National Medical College, Kolkata-700014. Ethics committee clearance was obtained from the mother institute.

\section{MATERIALS AND METHODS}

This was a descriptive comparative study with a simultaneous comparison group. The study was done in Calcutta National Medical College, Kolkata-14. A total number of 50 patients with at least 3 skin tags aged 30-50 years and who are not established diabetic were included in the study. 50 individuals aged between 30-50 years whose age and sex was matched with the cases were examined in Dermatology Outpatient Department in Calcutta National Medical College served as comparisons for the study. The fasting blood glucose, HbA1c, cholesterol, HDL, LDL, VLDL, triglyceride levels and BMI (basal metabolic rate) were measured for both study groups. Statistical analysis was done by SPSS Version 16.

\section{RESULTS}

There was significant statistical difference in fasting blood glucose, glycosylated haemoglobin, high-density lipoprotein, lowdensity lipoprotein, very low-density lipoprotein, cholesterol, triglyceride and basal metabolic rate between the study group and the comparison group. The patients' group showed significantly higher levels of all the above parameters when compared with the comparison group ( $\mathrm{p}$ value $<0.001$ ).

\section{CONCLUSION}

Blood glucose, cholesterol and triglyceride levels should be checked from time to time in patients with skin tags to provide corrective measures in the prevention and further exacerbation in presence of metabolic syndrome.

\section{KEYWORDS}

Metabolic Syndrome, Acrochordons, STs (Skin Tags), Body Mass Index (BMI).

HOW TO CITE THIS ARTICLE: De D, Biswas M, Pal D, et al. Can acrochordons be a marker of metabolic syndrome? J. Evolution Med. Dent. Sci. 2017;6(56):4183-4187, DOI: 10.14260/Jemds/2017/906.

\section{BACKGROUND}

Skin tags or acrochordons are small, soft, pedunculated, often pigmented lesions, usually occurring in the eyelids, neck and axillae. These tumours are flesh coloured and raised from the surface of the skin on a fleshy stalk called the peduncle. These tumours feel like soft bags. Usually, 2-3 in number, these are like the grain of rice. ${ }^{(1,2)}$ But, they may range in size from 1-2 $\mathrm{mm}$ papules in the eyelids to $1-2 \mathrm{~cm}$ baggy polyps on the trunk.(3) The surface of a skin tag maybe smooth or irregular in appearance. The most common site of skin tag is on the sides of the neck where they may be mixed with typical small,

Financial or Other, Competing Interest: None.

Submission 08-05-2017, Peer Review 03-07-2017,

Acceptance 08-07-2017, Published 13-07-2017.

Corresponding Author:

Dr. Devyani De,

Assistant Professor, Department of Biochemistry,

Flat No. $1 F$, Natural Top, 77A Christopher Road,

Kolkata-700046, West Bengal, India.

E-mail: devyanidedas@yahoo.co.in

DOI: $10.14260 /$ jemds $/ 2017 / 906$ sessile seborrheic keratosis.(4) These flesh coloured teardropshaped tags, feel like small bags. Based on twisted pedicle, they become inflamed, tender and even gangrenous. Both sexes are affected equally. Skin tags usually grow as we age. Increasing number of skin tags was seen with the increase in weight and pregnancy. Skin tags may occur at unusual sites of the body. A huge skin tag have been described on the penis.(5) A skin tag on the glans penis unassociated with condom catheter use has also been described.(2) Skin tags of the oral mucosa, anus and vulvovaginal areas may be found.(6) The symptoms are asymptomatic. Sometimes, they become painful due to irritation or torsion.(3) Sometimes, pruritus or discomfort occur when an acrochordon is snagged by jewellery or clothing.(6) There are many studies where an association have been found between acrochordons with conditions such as obesity, diabetes mellitus, atherogenic lipid profile, acromegaly and Crohn's disease.(7) About 30\% and $65 \%$ of adults were found overweight, obese or having abdominal obesity in various surveys in urban India.(8) The factors, which contribute for diabetes, dyslipidaemia and cardiovascular disease are abdominal obesity and insulin resistance. $(9,10)$ Although, many disorders are considered to 
be accompanied by skin tags, no sufficient studies have been conducted to assay the real risk. This survey was designed to evaluate the risk of dyslipidaemia in people with acrochordons in normal population. Along with these, the addition of fast foods and sedentary lifestyle with no exercise in this busy world needs due mention in the increase of obesity.

\section{Aims and Objectives}

1. Can acrochordons be a marker of metabolic syndrome?

2. To find out the association of lipid profile, fasting blood glucose, HbA1c, lipid profile and BMI with acrochordons.

\section{MATERIALS AND METHODS \\ Study Design}

This was a descriptive comparative study with a simultaneous comparison group. The study was conducted in Calcutta National Medical College, Kolkata, during July 2016 to December 2016.

\section{Duration of Study}

6 months (July to December 2016).

\section{Sample Size}

50 cases with acrochordons was finally included and 50 agesex matched individuals who did not have acrochordons, but had come to the Department of Dermatology with other skin problems were included in the comparison group.

\section{Sample Size Determination}

All cases of acrochordons who attended the Department of Dermatology were included in the study after obtaining their informed consent. In this way, 50 cases with acrochordons could be detected during the study period and thus the sample size came about to 50. Comparison group was selected after matching age and sex with cases who attended Dermatology OPD with other skin problems, but not having acrochordons and who met the inclusion criteria.

\section{Selection of Cases and Comparison Group} Inclusion Criteria

1. All patients, both cases and comparison group age-sex matched were taken from the Department of Dermatology, Calcutta National Medical College, Kolkata14.

2. Patients with at least 3 skin tags were included in the study and served as cases. A skin tag is a soft, skincoloured growth that hangs from the skin on a thin piece of tissue called a stalk. A skin tag is defined as a furrowed, pedunculated skin coloured papule approximately $2 \mathrm{~mm}$ in width and 3-6 $\mathrm{mm}$ in height with a duration of lesions of at least 6 months duration.

3. All patients within the age of 30-50 years of age.

4. Patients were not established diabetic.

5. Individuals who attended the Department of Dermatology with some other dermatological problems, but did not have skin tags or acrochordons served as comparison group. They were examined by the doctors in the Outpatient Department of Dermatology and after being examined free from skin tags or acrochordons were included to be the comparison for the study. They were in the age group of 30-50 years and their age and sex were matched with the cases. The comparison group were nondiabetic persons without acrochordons or skin tags, but having other skin problems who visited the Department of Dermatology during the study period.

\section{Exclusion Criteria}

1. Pregnant women, patients with conditions causing fluid retention like heart failure, renal disease (failure) and decompensated liver disease, patients with neurological conditions, thyromegaly were excluded. These interfere with measurement of anthropometric features.

2. Patients who are known diabetic and receiving antidiabetic drugs.

3. Those persons who are having drugs with a known hyperlipidaemic effect.

4. Hypertensive patients on antihypertensive drugs, which have antihypertensive effect.

\section{Measurement of Various Anthropometric Parameters}

Blood pressure, height, weight, waist circumference, hip circumference and body mass index.

Measurement of biochemical parameters- Fasting blood glucose, HbA1c, serum HDL, serum LDL, serum cholesterol and serum triglyceride was analysed. Low-Density Lipoprotein (LDL) cholesterol and Very Low-Density Lipoprotein (VLDL) cholesterol values were calculated according to the following formulae- a) Very low-density lipoprotein cholesterol $=$ triglyceride/5. b) Low-density lipoprotein cholesterol $=$ cholesterol- (very low-density lipoprotein cholesterol + high-density lipoprotein cholesterol). Blood was collected after twelve hours of fasting. Blood collection was done after each patient and comparison was thoroughly examined in the Department of Dermatology after fulfilling the criteria required for the study and comparison groups. All these parameters were measured by autoanalyzer in the Department of Biochemistry, Calcutta National Medical College, Kolkata-14. BMI (basal metabolic index) was calculated by dividing body weight to height square $(\mathrm{kg} / \mathrm{m} 2)$. Patients were considered according to their BMI- BMI $\leq 18$ as thin, BMI between 19 and 25 as normal, BMI between 26 and 29 as overweight, BMI $\geq 30$ as obese. The height of patients was measured by a measuring tape. The measurement was approximated to the nearest $0.5 \mathrm{~cm}$. The weight of the cases and comparison were measured by a weight measuring device. The patient and the comparison groups were informed nicely about the aim and procedure of the study. Only willing patients and subjects for comparison were included in the study after having their informed consent.

\section{Statistical Analysis}

Data collected was analysed by SPSS (statistical package for social scientist) version 16 . The results were expressed as mean \pm SD values. The data was recorded in Microsoft excel and analysed using SPSS software (version 16). The significance of the difference between the groups was assessed by Student's t-test (two tailed, independent) to find the significance of the study parameters between the cases and the comparison groups. 


\section{RESULTS}

A total of 50 patients (cases) with at least 3 skin tags and age between 30-50 years from the Outpatient Department of Dermatology, Calcutta National Medical College, Kolkata-14 were included in the study. 50 comparisons were also selected for the study after being examined in the Department of Dermatology, Calcutta National Medical College, Kolkata-14. The study was conducted in a period of six months. Patients showed significantly higher levels of glucose $(p$ value $=0.000)$ and HbA1c $(p$ value $=0.04)$ levels Statistically significant $p$ values ( $p$ value $=0.000$ ) were also found in cholesterol, high-density lipoprotein, low-density lipoprotein, very low-density lipoprotein and triglyceride levels in the study group than in the comparison group. All patients also showed significantly higher body mass index when compared to comparison group ( $p$ value $=0.000$ ).

\begin{tabular}{|c|c|c|c|c|c|}
\hline Parameter & Case (mean \pm SD) & Comparison Group (mean \pm SD) & t-value & P value & Statistical Significance \\
\hline Glucose & $114.92 \pm 10.07$ & $83.44 \pm 7.30$ & 17.87 & 0.000 & Significant \\
\hline HbA1c & $6.5780 \pm 60$ & $4.85 \pm 0.53$ & 2.06 & 0.04 & Significant \\
\hline Cholesterol & $232.35 \pm 38.19$ & $146.32 \pm 24.01$ & 13.48 & 0.000 & Significant \\
\hline HDL & $31.94 \pm 4.51$ & $48.62 \pm 8.03$ & 12.87 & 0.000 & Significant \\
\hline LDL & $143.70 \pm 11.35$ & $116.56 \pm 8.22$ & 13.69 & 0.000 & Significant \\
\hline VLDL & $45.34 \pm 13.02$ & $23.71 \pm 7.19$ & 10.28 & 0.000 & Significant \\
\hline TG & $226.72 \pm 65.12$ & $118.54 \pm 35.93$ & 10.29 & 0.000 & Significant \\
\hline BMI & $33.45 \pm 3.94$ & $25.22 \pm 1.51$ & 13.88 & 0.000 & Significant \\
\hline \multicolumn{7}{|l}{} \\
\hline
\end{tabular}

\begin{tabular}{|c|c|c|c|}
\hline \multirow{2}{*}{ Acrochordon } & \multicolumn{2}{|c|}{ Metabolic Syndrome } & \multirow{2}{*}{ Total } \\
\cline { 2 - 3 } & Present & Absent & \\
\hline Present & $34(68.0)$ & $16(32.0)$ & 50 \\
\hline Absent & $19(38.0)$ & $31(62.0)$ & 50 \\
\hline Total & $\mathbf{5 3}$ & $\mathbf{4 7}$ & $\mathbf{1 0 0}$ \\
\hline \multicolumn{2}{|c|}{$\begin{array}{r}\text { Table 2. Association Between Presence } \\
\text { of Acrochordon and Metabolic Syndrome }\end{array}$} \\
\hline
\end{tabular}

Comment- Metabolic syndrome was found to be significantly associated with the presence of acrochordons $\left(\chi^{2}\right.$ (1) $=9.03, p=0.003$ ).

$\mathrm{OR}=3.47(95 \%, \mathrm{CI}=1.41-8.64)$

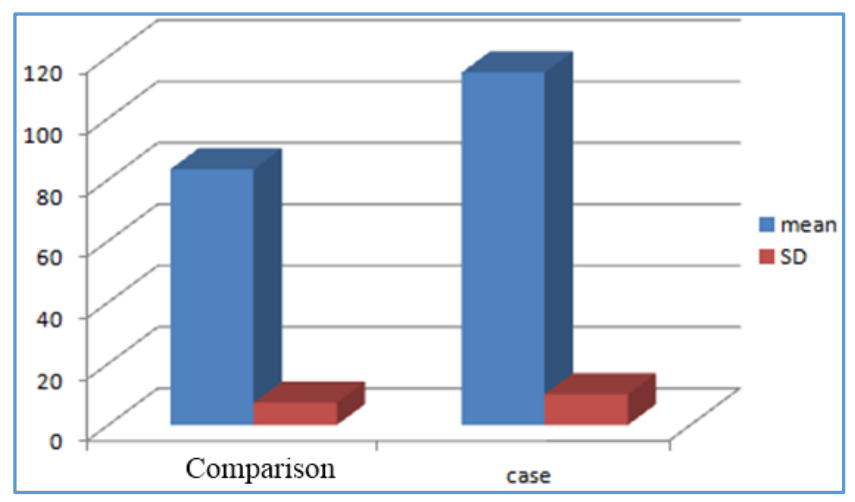

Figure 1. Showing Glucose Levels

(Y Axis) in Comparison and Cases

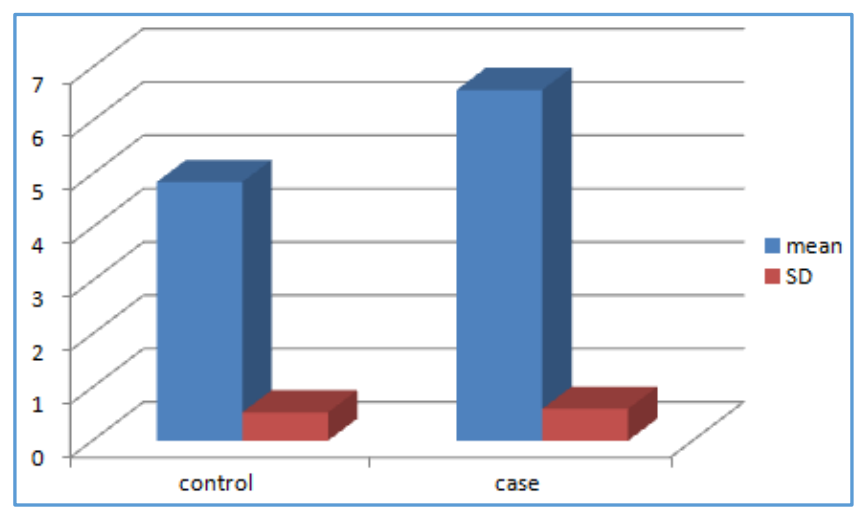

Figure 2. Showing HbA1c Levels (Y Axis) in Comparison and Cases

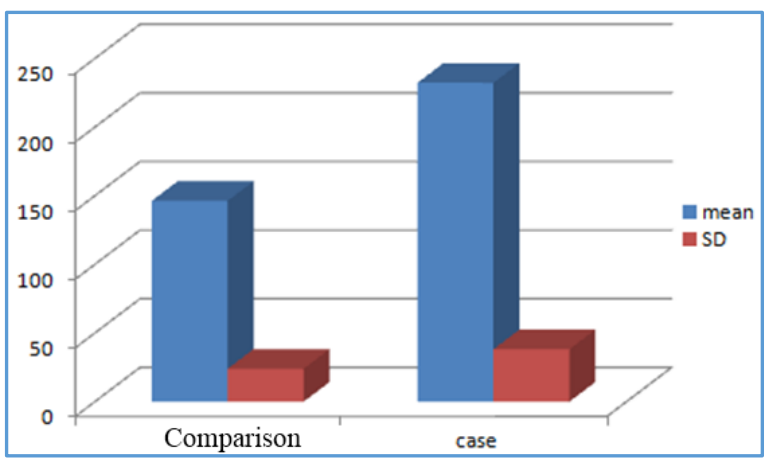

Figure 3. Showing Total Cholesterol Levels (Y Axis) in Comparison and Cases

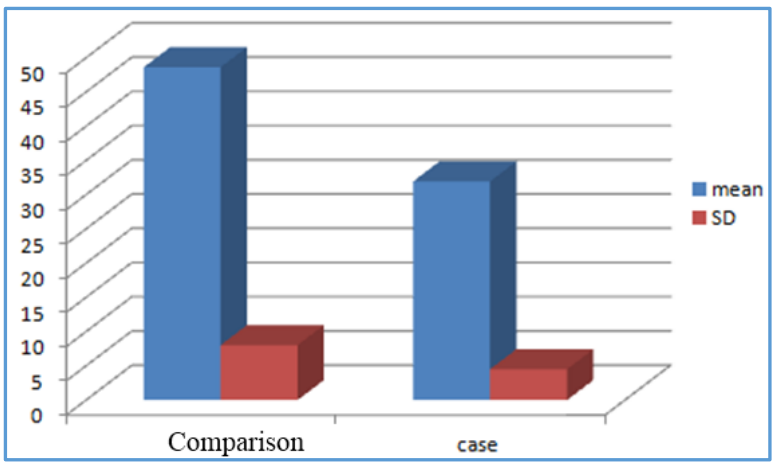

Figure 4. Showing High-Density Lipoprotein Levels (Y Axis) in Comparison and Cases

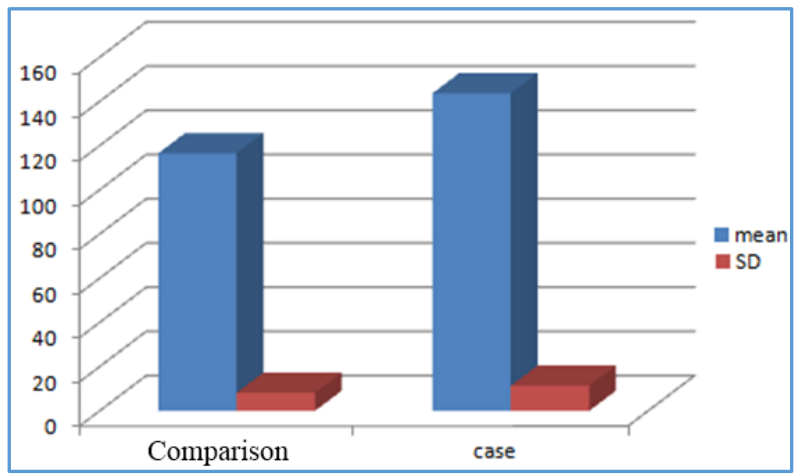

Figure 5. Showing Low-Density Lipoprotein Levels (Y Axis) in Comparison and Cases 


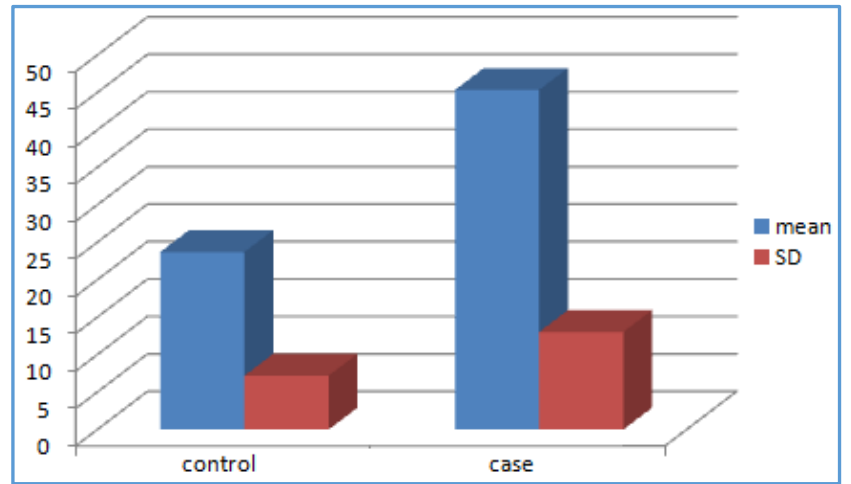

Figure 6. Showing Very Low-Density Lipoprotein Levels (Y Axis) in Comparison and Cases

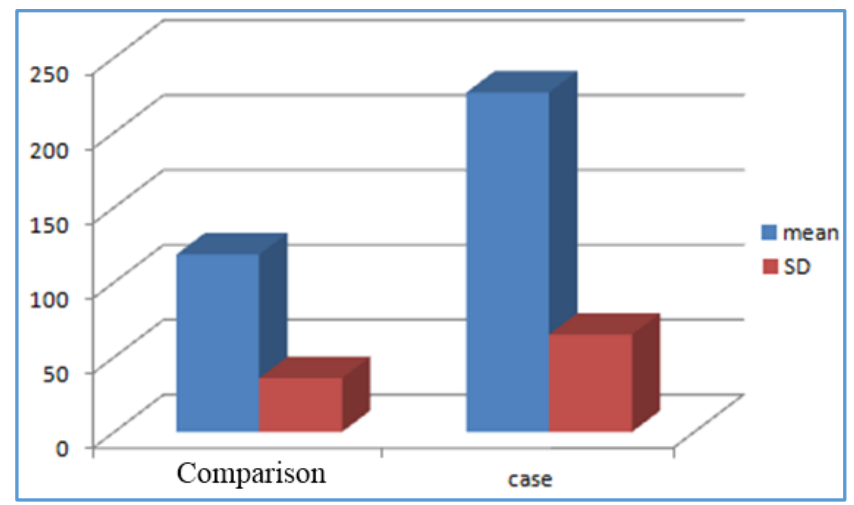

Figure 7. Showing Low Triglyceride Levels (Y Axis) in Comparison and Cases

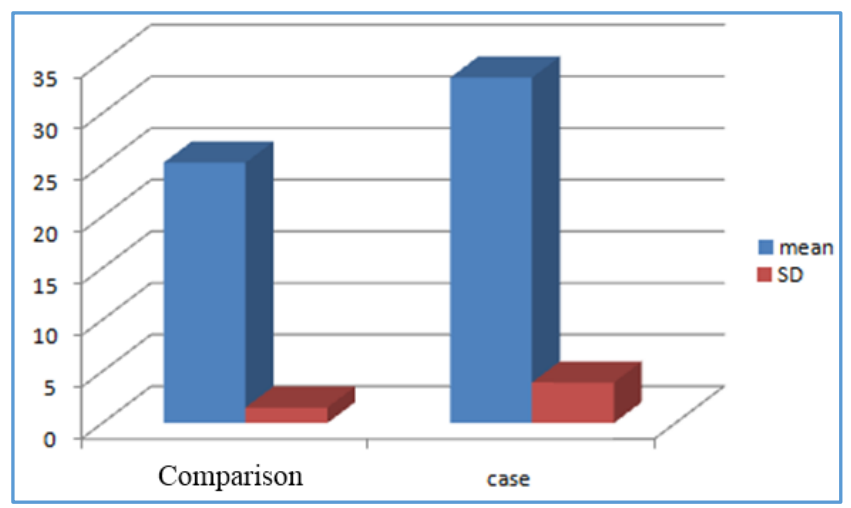

Figure 8. Showing Body Mass Index Levels (Y Axis) in Comparison and Cases

\section{DISCUSSION}

In the present study, we found that there is a positive correlation between metabolic syndrome and skin acrochordons. The glucose $(\mathrm{p}=0.000)$ and $\operatorname{HbA1c}(\mathrm{p}=0.04)$ levels were significantly higher in patients with STs and were statistically significant. Previous studies have found a positive correlation between STs and diabetes mellitus.(11,12) A recent study(6) also showed an increased risk of diabetes mellitus in patients with STs. Bahgat and Safory ${ }^{(13)}$ also found a positive relationship between STs and diabetes mellitus. We also found a positive correlation between lipid profile and skin tags (acrochordons). The ' $p$ ' value of all the parameters done was very significant. Erdogan et al found increased total cholesterol in patients with skin tags.(7) Gorpelioglu C et al found total cholesterol levels with low-density lipoprotein serum levels were higher in patients with skin tags when compared with comparison group. The above two studies meet the results of the present study ( $\mathrm{p}$ value $=0.000$ ) Another study conducted by Sari et al also complies with our results.(14) A recent study(15) found that mean total cholesterol levels and total cholesterol/HDL ratio were significantly higher in the cases than those in the comparison group. A study conducted by Wali and Wali(16) also found that high value of plasma lipids are associated with skin tags. The glucose and HbA1c levels were higher in patients with the STs and were statistically significant. BMI was also higher in patients with STs and was statistically significant $(\mathrm{p}=0.000)$. In some studies, association between BMI and ST(17,18) was found. In another study, no association was found.(11) They concluded that epidermal growth factor or other growth factors may play a role in the pathogenesis of skin tags. Several other studies conducted by Erdogan et al,(7) Demir S and Demir $Y,{ }^{(19)}$ Sari et al(14) has findings which fully correlate with our findings. As we have found here, it was also found in earlier studies that multiple skin tags are frequently associated with non-insulin-dependent diabetes mellitus and obesity.(20)

\section{CONCLUSION}

The data in our study revealed that acrochordons are really a marker of metabolic syndrome. Patients with acrochordons had significantly high glucose levels, HbA1c levels and lipid profile. The basal metabolic index was found to be significantly high in the patient group. Change of lifestyle, regular physical exercise, balanced diet, changing from carbohydrate to protein diet along with avoidance of sedentary lifestyle will definitely reduce metabolic syndrome in patients with hyperlipidaemia, skin tags and metabolic syndrome. Due to sedentary lifestyle, increase in weight and obesity is a leading problem in developing and developed countries. Weight reduction should be taken into account seriously in such patients and due care should be taken to attain the expected weight for height as early as possible. Diet rich in polyunsaturated fatty acids is suggested to avoid coronary problems. Our study had a limited number of patients and comparisons from a single population. Further studies with larger patient and comparison groups from different populations is definitely required in further bringing out the relationship between skin tags and metabolic syndrome.

\section{ACKNOWLEDGEMENT}

The authors gratefully acknowledge the help and support provided by the respective departments in carrying out the study. The persons who served as patient and comparison group in the study needs special thanks without whose participation this study would not have come into being.

\section{REFERENCES}

[1] James WD, Berger TG, Elston DM. Chapter 29. Andrews' disease of skin: clinical dermatology. Epidermal nevi, neoplasms and cysts. $11^{\text {th }}$ edn. 2011;601-2.

[2] Allegue F, Fachal C, Perez-Perez L. Friction induced skin tags. Dermatol Online J 2008;14(3):18.

[3] Bolognia JL, Jorizzo JL, Rapini RP. In: Kamino H, Meena S, Pui J. eds. Neoplasm of skin. Second skin Dermatology. 2nd edn 2008;18:1813. 
[4] Bums T, Breathnach SM, Cox NH, et al. Non-melanoma skin cancer and other epidermal skin tumours. Rook's Textbook of Dermatology. $8^{\text {th }}$ edn. 2010;3:52.4052.41.

[5] El Safoury 0, Rashid L, Ibrahim M. A study of androgen and oestrogen receptors alpha, beta in skin tags. Indian J Dermatol 2010;55(1):20-4.

[6] Rasi A, Soltani- Arabshahi R, Shahbazi N. Skin tag as a cutaneous marker for impaired carbohydrate metabolism: a case control study. Int J Dermatol 2007;46(11):1155-9.

[7] Erdogan BS, Aktan S, Rota S, et al. Skin tags and atherosclerotic risk factors. J Dermatol 2005;32(5):371-5.

[8] Misra A, Khurana L. Obesity and the metabolic syndrome in developing countries. J Clin Endocrinol Metab 2008:93(11 Suppl 1):S9-30.

[9] Hamdy 0, Porramatikul S, Al-Ozairi E. Metabolic obesity: the paradox between visceral and subcutaneous fat. Curr Diab Rev 2006:2(4):367-73.

[10] Premanath M, Basavanagowdappa H, Mahesh M, et al. Correlation of abdominal adiposity with components of metabolic syndrome, anthropometric parameters and insulin resistance, in obese and non-obese, diabetics and non-diabetics: a cross-sectional observational study. (Mysore Visceral Adiposity in Diabetes Study). Indian J Endocrinol Metab 2014;18(5):676-82.

[11] Kahana M, Grossman E, Feinstein A, et al. Skin tags: a cutaneous marker for diabetes mellitus. Acta Derma Venerol 1987;67(2):175-7.
[12] Agarwal JK, Nigam PK. Acrochordon: a cutaneous sign of carbohydrate intolerance. Australas J Dermatol 1987;28(3):132-3.

[13] Bosseila M, Shaker O. The tissue expression of insulin like growth factor (IGF-1) in acrochordons. J Egypt Women's Dermatol Soc 2007;57-62.

[14] Sari R, Akman A, Alpsoy E, et al. The metabolic profile in patients with skin tags. Clin Exp Med 2010;10(3):193-7.

[15] Idris S, Sunitha S. Assessment of BMI, Serum leptin levels and lipid profile in patients with skin tags. J Clin Diagn Res 2014;8(9):CC01-3.

[16] Wali V, Wali VV. Assessment of various biochemical parameters and BMI in patients with skin tags. Journal of Clinical and Diagnostic Research 2016;10(1):BC09BC11.

[17] Gorpelioglu C, Erdal E, Ardicoglu Y, et al. Serum leptin atherogenic lipids and glucose levels in patients with skin tags. Indian J Dermatol 2009;54(1):20-2.

[18] Bhargava P, Mathur SK, Mathur DK, et al. Acrochordon, diabetes and associations. Indian Dermatol Venerol Leprol 1996;62(4):226-8.

[19] Demir S, Demir Y. Acrochordon and impaired carbohydrate metabolism. Acta Diabetol 2002;39(2):57-9.

[20] Austin MA, King MC, Vranizan KM, et al. Atherogenic lipoprotein phenotype. A proposed genetic marker for coronary heart disease risk. Circulation 1990;82(2): 495-506. 